\title{
ON EXTENSIONS OF PSEUDO-INTEGERS
}

\author{
HEATHER RIES
}

\begin{abstract}
An abelian group $A$ is pseudofree of rank $k$ if the $p$-localization of $A$ is isomorphic to the $p$-localization of $\mathbb{Z}^{k}$ for all primes $p$, i.e. $A_{p} \cong \mathbb{Z}_{p}^{k}$ for all primes $p$. If $k=1$, we call $A$ a group of pseudo-integers. We may assume, in this case, that $\mathbb{Z} \subseteq A \subset \mathbb{Q}$ and that for any prime $p_{i}$ there is a maximal exponent $r_{i}$ so that $\frac{\frac{1}{s_{i}}}{p_{i}^{2}} \in A$. The group $A$ is then generated by $\left\{\frac{1}{p_{i}^{r i}} \mid p_{i}\right.$ is a prime $\}$ and we write $A=\left\langle\frac{1}{p_{i}^{r_{2}}}\right\rangle$. We say a pseudofree group of rank $k$ is completely decomposable if it can be written as the direct sum of $k$ groups of pseudo-integers.

If $A$ is pscudofree of rank $k, B$ is pseudofree of rank $l$, and $B \longmapsto E \rightarrow A$ is an extension in $\operatorname{Ext}(A, B)$ then $E$ must be pseudofree of rank $k+l$. In this paper, we consider $\operatorname{Ext}(\bar{P}, P)$ for $P=\left\langle\frac{1}{p_{i}^{2 i}}\right\rangle$ and $\bar{P}=\left\langle\frac{1}{p_{i}^{r i}}\right\rangle$ groups of pseudo-integers. We determine when it is possible, in terms of the defining exponents of $P$ and $\bar{P}$, for $\operatorname{Ext}(\bar{P}, P)$ to contain certain extensions (which we'll call nontrivial) where $E$ is completely decomposable as a pseudofree group of rank 2. We find that $\operatorname{Ext}(\bar{P}, P)$ contains such extensions if and only if $r_{i} \leq \bar{r}_{i}$ almost everywhere and $r_{i}<\bar{r}_{i}$ for an infinite number of primes.
\end{abstract}

\section{Introduction.}

In [1] Casacuberta and Hilton introduce the concept of the extended genus of a nilpotent group $N$, denoted $\operatorname{EG}(N)$. It is defined to be the set of isomorphism classes of nilpotent groups $M$ so that the $p$-localizations of $M$ and $N$ are isomorphic for all primes $p$, i.e. $M_{p} \cong N_{p}$ for all primes $p$. If $A$ is a finitely-generated abelian group, they show that to sludy $\operatorname{EG}(A)$ it is only necessary to examine $\mathrm{EG}\left(\mathbb{Z}^{k}\right)$ where $k$ is the torsionfree rank of $A$. The exiended genus of $\mathbb{Z}$ is completely described in [2]. There it is indicated that if $A \in \mathrm{EG}(\mathbb{Z})$ then $A$ is similar in many ways to $\mathbb{Z}$ and hence $A$ is called a group of pseudo-integers. We will adopt this terminology and, accordingly, if $A \in E G\left(\mathbb{Z}^{k}\right)$ we will say that $A$ is pseudofree of rank $k$. As in [1], we define a pseudofree group 
$A$ of rank $k$ to be completely decomposable if it is the direct sum of $k$ groups of pseudo-integers, i.e. $A=\bigoplus_{i=1}^{k} A_{i}$ where each $A_{2}$ is a group of pseudo-integers.

In this paper, we investigate $\operatorname{Ext}(\bar{P}, P)$ for $P$ and $\bar{P}$ groups of pseudointegers. In $[5]$, the structure of $\operatorname{Hom}(\bar{P}, P)$ and $\operatorname{Ext}(\ddot{P}, P)$ as abclian groups is identified, while in [4] it is shown that, for certain $\bar{P}$ and $P$; $\operatorname{Ext}(\bar{P}, P)$ contains extensions $P \hookrightarrow E \rightarrow \bar{P}$ where $E$ is not completely decomposable as a pseudofree group of rank 2 . Here we should note that for any extension $P \leadsto E \rightarrow \bar{P}$ of $\bar{P}$ by $P, E$ must be pseudofree of rank 2 since $p$-localization is exact and $\mathbb{Z}_{p}$ is a p.i.d. In this paper, we determine when it is possible for $\operatorname{Ext}(\tilde{P}, P)$ to contain extensions $P \rightarrow E \rightarrow \bar{P}$ where $E$ is a certain type of completely decomposable pseudofrec group of rank 2, i.e. $E \cong B_{1} \oplus B_{2}$ for particular groups of pseudo-integers $B_{1}$ and $B_{2}$.

In Section 1, we define the type of completely decomposable extension (which we will call nontrivial) that we hope to find by making clear our restrictions on $B_{1}$ and $B_{2}$. We describe, in Section 2 , when $P$ embeds into $B_{1} \oplus B_{2}$ for arbitrary groups of pseudo-integers $P, B_{1}$, and $B_{2}$. Since we want the quotient resulting from such an embedding to bc isomorphic to $\bar{P}$ (and hence torsionfree), in Section 3 we characterize the torsion subgroup of the quotient resulting from a given embedding of $P$ into $B_{1} \oplus B_{2}$. Finally, in Section 4, we have a theorem which allows us to determine when such a quotient is in fact isomorphic to $\bar{P}$. We then find exactly when $\operatorname{Ext}(\dot{P}, P)$ contains nontrivial completely decomposable extensions in terms of defining characteristics of $\vec{P}$ and $P$.

These results were completed as part of my dissertation at SUNYBinghamton. I would like to express my deepest appreciation to my advisor, Professor Peter Hilton, for his invaluable guidance and constant encouragement.

\section{Pseudo-Integers and Extensions.}

For $A, B$ abelian groups, we state an interpretation of $\operatorname{Ext}(A, B)$ which may be found in [3]. The extensions $B \neg E_{1} \rightarrow A$ and $B \neg E_{2} \rightarrow A$ are said to be equivalent if there exists $\psi: E_{1} \rightarrow E_{2}$ so that the following diagram commutes:

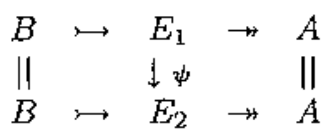

Since $\psi$ is necessarily an isomorphism, the above relation is an equivalence relation and $\operatorname{Ext}(A, B)$ is regarded as the sct of equivalence classes of extensions. It may be shown to have an abelian group structure with 
zero element the equivalence class of the extension $B \mapsto B \oplus A \rightarrow A$ where $B$ cmbeds naturally into $B \oplus A$ and $B \oplus A$ projects naturally onto A.

In this paper we shall be concerned with certain extensions of the form $P: E \rightarrow \ddot{P}$ where $P$ and $\bar{P}$ are groups of pseudo-integers and $E$ is necessarily pseudofree of rank 2 . However, before we consider particular extensions in $\operatorname{Ext}(\ddot{P}, P)$ we will note some results previously obtained on the algebraic structure of $\operatorname{Hom}(\bar{P}, P)$ and $\operatorname{Ext}\left(\tilde{P}_{:} P\right)$ as abelian groups.

We first introduce notation to be used throughout the paper. In [2] it is demonstrated that if $P$ is a group of pseudo-integers then we may assume $\mathbb{Z} \subseteq P \subseteq Q$ and for any prime $p_{i}$ there is a maximal exponent $r_{i} \geq 0$ so that $\frac{1}{p_{i}^{r_{2}}} \in P$. Moreover, as may be easily shown, $P$ is then generated by the set $\left\{\frac{1}{p_{i}^{p_{i}}} \mid p_{i}\right.$ is a prime $\}$ and the elements of $P$ are represented by reduced fractions $\frac{a}{b}$ where $b$ has prime power factors $p_{i}^{l_{2}}$, with $l_{i} \leq r_{i}$. We will henceforth denote $P$ by $\left\langle\frac{1}{p_{i}^{p_{i}}}\right\rangle$ and assume all fractions mentioned to be reduced.

We will often employ the following result (also from [2]) concerning the isomorphism problem for groups of pseudo-integers.

Theorem. Assume $P=\left\langle\frac{1}{p_{i}^{r_{i}}}\right\rangle$ and $H=\left\langle\frac{1}{p_{i}^{k_{i}}}\right\rangle$ are groups of pseudointegers. Then $P \cong H$ if and only if $r_{i}=k_{i}$ almost everywhere.

Now suppose $P=\left\langle\frac{1}{p_{i}^{r_{T}}}\right\rangle$ and $\bar{P}=\left\langle\frac{1}{p_{i}^{\gamma_{i}}}\right\rangle$ are groups of pseudo-integers. The above theorem implies that to determine $\operatorname{Hom}(\bar{P}, P)$ one need only consider the case where $\bar{r}_{i} \geq r_{i}$ for infinitely many $i$ and the case where $\bar{r}_{i} \leq r_{i}$ everywhere. The following results are then obtained in [5] which show that $\operatorname{Hom}(\bar{P}, P)$ is either trivial or another group of pseudointegers.

Theorem. If $\bar{r}_{i}>r_{i}$ for infinitely many $i$ then $\operatorname{Hom}(P, P)=0$.

Theorem. If $\bar{r}_{i} \leq r_{i}$ for all $i$ then $\operatorname{Hom}(\bar{P}, P\rangle \cong\left\langle\frac{1}{p_{i}^{l_{i}}}\right\rangle$ where $l_{i}=$ $r_{i}-\ddot{r}_{i}$.

A special case of a theorem proved for pseudofree groups of arbitrary rank then describes $\operatorname{Ext}(\bar{P}, P)$.

Theorem. Suppose $\bar{P}$ and $P$ are groups of pseudo-integers. Then $\operatorname{Ext}(\bar{P}, P) \cong V \oplus(\mathbb{Q} / \mathbb{Z})^{1-l}$ where $V$ is a $\mathbb{Q}$-vector space of rank c and $l$ is the rank of $\operatorname{Hom}(\bar{P}, P)$. 
In this paper, we shall consider extensions $P \hookrightarrow E \rightarrow \bar{P}$ in $\operatorname{Ext}(\bar{P}, P)$ where $E$ is completely decomposable as a pseudofree group of rank 2, i.e. $E \cong B_{1} \oplus B_{2}$ for $B_{1}$ and $B_{2}$ groups of pseudo-integers. We will sometimes refer to $E$ itself as the extension. Now it is clear that one such extension is always present, i.e. the zero extension $P \mapsto P \oplus \bar{P} \rightarrow \bar{P}$ in $\operatorname{Ext}(\bar{P}, P)$. So we wish to determine when $\operatorname{Ext}(\bar{P}, P)$ contains other, less obvious completely decomposable extensions. We therefore define a completely decomposable extension $P \mapsto B_{1} \oplus B_{2} \rightarrow \bar{P}$ to be trivial if $P \cong B_{1}, \bar{P} \cong B_{2}$ or $P \cong B_{2}, \bar{P} \cong B_{1}$ and nontrivial otherwise. As we see from the following theorem, our definition guarantees that a nontrivial extension will not belong to the zero element of $\operatorname{Ext}(\bar{P}, P)$. Hence our goal will be to describe when $\operatorname{Ext}(\ddot{P}, P)$ contains nontrivial completely decomposable extensions.

Theorem 1.1. If $P \leadsto B_{1} \oplus B_{2} \rightarrow \bar{P}$ is nontrivial in $E_{x} t(\bar{P}, P)$ then the extension is also nonzero in $\operatorname{Ext}(\bar{P}, P)$.

Proof: Suppose $P \mapsto B_{1} \ominus B_{2} \rightarrow \bar{P}$ does belong to the zero element in $\operatorname{Ext}(\bar{P}, P)$. This implies that $P \hookrightarrow B_{1} \ominus B_{2} \rightarrow \bar{P}$ is equivalent to the natural extension $P \rightarrow P \oplus \bar{P} \rightarrow \bar{P}$. Hence $B_{1} \ominus B_{2}$ is necessarily isomorphic to $P \oplus P$. Now in [4] it is shown that the decomposition of a pseudofree group of rank 2 must be unique, i.e. if $P \oplus \bar{P} \cong B_{1} \ominus B_{2}$ then $P \cong B_{1}, \vec{P} \cong B_{2}$ or $P \cong B_{2}, \vec{P} \cong B_{1}$. Thus we have a contradiction since we assumed our extension to be nontrivial.

\section{Embeddings.}

Since we wish to find extensions of the form $P \mapsto B_{1} \oplus B_{2} \rightarrow \bar{P}$, we must first determine when it is possible to embed $P$ into $B_{1} \oplus B_{2}$. We note that since $P, B_{1}, B_{2}$ are subgroups of $\mathbb{Q}$, to describe an embedding of $P$ into $B_{1} \oplus B_{2}$ we necd only indicate the ordered pair to which $1 \in P$ is sent. For, if $I \mapsto\left(\frac{u_{1}}{v_{1}}, \frac{u_{2}}{v_{2}}\right)$ in $B_{1} \oplus B_{2}$ then $\frac{a}{b} \mapsto\left(\frac{u_{1} \frac{a}{b}}{v_{1}}, \frac{u_{2} a}{v_{2} b}\right)$ for any $\frac{a}{b} \in P$.

We shall consider only embeddings of the form $1 \mapsto\left(\frac{u_{2}}{v_{1}}, \frac{u_{2}}{v_{2}}\right)$ where $u_{1} \neq 0$ and $u_{2} \neq 0$ as otherwise, either the quotient has torsion (and hence could not be isomorphic to $\bar{P}$ ) or we are led to a trivial extension. For suppose that $P \longmapsto B_{1} \oplus B_{2} \rightarrow \bar{P}$ is an extension in $\operatorname{Ext}(\bar{P}, P)$ so that $P$ embeds into $B_{1} \oplus B_{2}$ with the embedding $1 \mapsto\left(\frac{u_{1}}{v_{1}}, 0\right)$ for some $u_{1} \neq 0$. Now $\frac{B_{1} \Phi B_{2}}{P} \cong B_{1} / P \oplus B_{2}$ and $\frac{B_{1} \oplus B_{2}}{P} \cong \ddot{P}$. But $B_{1} / P$ is a torsion group and hence must be trivial which indicates that $B_{1} \cong P$. Now $B_{2}$ must then be isomorphic to $\bar{P}$ and we see that the extension is trivial. Of course an embedding of the form $1 \mapsto\left(0, \frac{u_{2}}{v_{2}}\right)$ for some $u_{2} \neq 0$ yields a similar result. 
The next two theorems address the problem of embedding $P$ into $B_{1} \oplus$ $B_{2}$. The first describes when a specific homomorphism from $P$ to $\mathbb{Q} \oplus \mathbb{Q}$ is an embedding and the second shows when it is possible, in terms of the exponents of $P, B_{1}$, and $B_{2}$, to embed $P$ into $B_{1} \oplus B_{2}$. For $n \in \mathbb{Z}$ and $p_{2}$ a. prime, let $v_{p_{i}}(n)$ be the usual $p_{i}$-valuation of $n$, i.e. the highest power of $p_{i}$ that divides $n$. Then for $\frac{u}{v}$ in $\mathbb{Q}$, we define $v_{p_{i}}\left(\frac{u}{v}\right)=v_{p_{i}}(u)-v_{p_{i}}(v)$. Finally, let $P=\left\langle\frac{1}{p_{2}^{\gamma_{i}}}\right\rangle, B_{1}=\left\langle\frac{1}{p_{i}^{m_{i}}}\right\rangle$, and $B_{2}=\left\langle\frac{1}{p_{i}^{n_{i}}}\right\rangle$ represent the groups of pseudo-integers.

Theorem 2.1. The embedding of $P$ into $\mathbb{Q} \oplus \mathbb{Q}$ defined by $1 \mapsto$ $\left(\frac{u_{1}}{v_{1}}, \frac{u_{2}}{v_{2}}\right)$ is an embedding of $P$ into $B_{1} \oplus B_{2}$ if and only if $r_{i}-v_{p_{i}}\left(\frac{u_{1}}{v_{1}}\right) \leq m_{i}$ and $r_{i}-v_{p_{2}}\left(\frac{u_{2}}{v_{2}}\right) \leq n_{i}$ for all $i$.

Proof: Now $1 \mapsto\left(\frac{u_{1}}{v_{1}}, \frac{u_{2}}{v_{2}}\right)$ restricts to an embedding of $P$ into $B_{1} \oplus B_{2}$ if and only if $\frac{1}{p_{i}^{r_{i}}} \mapsto\left(\frac{u_{1}}{v_{1} p_{i}^{r_{i}}}, \frac{u_{2}}{v_{2} p_{i}^{i}}\right) \in B_{1} \oplus B_{2}$ for all $i$. But $\frac{u_{1}}{v_{1} p_{i}^{i}} \in B_{1} \Longleftrightarrow$ $v_{p_{i}}\left(\frac{u_{1}}{v_{1} p_{i}^{r_{i}}}\right) \geq-m_{i} \Longleftrightarrow v_{p_{i}}\left(\frac{u_{1}}{v_{1}}\right)-r_{i} \geq-m_{i} \Longleftrightarrow r_{i}-v_{p_{i}}\left(\frac{u_{1}}{v_{1}}\right) \leq m_{i}$. Similarly, $\frac{u_{2}}{v_{2} p_{2}^{r_{i}}} \in B_{2} \Longleftrightarrow r_{i}-v_{p_{i}}\left(\frac{u_{2}}{v_{2}}\right) \leq n_{i}$.

Note that, in particular, $1 \mapsto(1,1)$ defines an embedding of $P$ into $B_{1} \oplus B_{2}$ if and only if $r_{i} \leq m_{i}$ and $r_{i} \leq n_{i}$ for all $i$.

Theorem 2.2. The group of pseudo-integers $P$ embeds into $B_{1} \ominus B_{2}$ if and only if $r_{i} \leq m_{i}$ and $r_{i} \leq n_{i}$ almost everywhere.

Proof: Assume $P$ embeds into $B_{1} \oplus B_{2}$ and let $1 \mapsto\left(\frac{u_{1}}{v_{1}}, \frac{u_{2}}{v_{2}}\right)$ be an embedding. Since $v_{p_{i}}\left(\frac{u_{1}}{v_{1}}\right)=0$ and $v_{p_{i}}\left(\frac{u_{2}}{v_{2}}\right)=0$ for all but a finite number of $i$, it is clear from Theorem 2.1 that $r_{i} \leq m_{i}, r_{i} \leq n_{i}$ almost everywhere.

If we suppose that $r_{i} \leq m_{i}$ and $r_{i} \leq n_{i}$ alnost everywhere, we can easily construct an embedding. Let $S_{1}=\left\{j \mid r_{j}>m_{j}\right\}$ and $S_{2}=$ $\left\{k \mid r_{k}>n_{k}\right\}$ and consider the embedding of $B$ into $\mathbb{Q} \oplus \mathbb{Q}$ defined by $1 \mapsto\left(\prod_{j \in S_{1}} p_{j}^{r_{j}-m_{3}}, \prod_{k \in S_{2}} p_{k}^{r_{k}-n_{k}}\right)$. It can be readily verified that $r_{2}-$ $v_{p_{i}}\left(\prod_{j \in S_{1}} p_{j}^{r_{j}-m_{j}}\right) \leq m_{i}$ and $r_{i}-v_{p_{i}}\left(\prod_{k \in S_{2}} p_{k}^{\tau_{k}-m_{k}}\right) \leq n_{i}$ for all $i$ and hence, by 'Theorem 2.1, we have an embedding of $P$ into $B_{1} \oplus B_{2}$.

\section{Torsion Subgroups of Quotients.}

Recall that our goal is to determine when it is possible to find nontrivial completely decomposable extcnsions in $\operatorname{Ext}(\bar{P}, P)$ i.e. extensions of the form $P \longmapsto B_{1} \oplus B_{2} \rightarrow \bar{P}$ where certain conditions are placed on $B_{1}, B_{2}$. Since, for such an extension to exist, the quotient $\frac{B_{1} \oplus B_{2}}{P}$ must 
be isomorphic to $\bar{P}$, we must determine when it is possible to embed $P$ into $B_{1} \oplus B_{2}$ so that the resulting quotient is torsionfree. Hence in this section we characterize the torsion subgroup of $\frac{B_{1} \Theta_{1} B_{2}}{P}$ with respect to a given embedding. This characterization, given in the next two theorems, is essential to our determination of the existence of completely decomposable extensions in $\operatorname{Ext}(\bar{P}, P)$. Given an embedding of $P$ into $B_{1} \oplus B_{2}$; we will use $Q$ to represent the resulting quotient of $B_{1} \ominus B_{2}$ by $P$, i.c. $Q=\frac{B_{1} \oplus B_{2}}{P}$. We will represent the torsion subgroup of this quoticnt by $T Q$. Also, we will assume $P=\left\langle\frac{1}{p_{i}^{p_{2}}}\right\rangle, B_{1}=\left\langle\frac{1}{p_{i}^{n_{i}}}\right\rangle$, and $B_{2}=\left\langle\frac{1}{p_{i}^{i_{i}}}\right\rangle$.

Theorem 3.1. Suppose $P$ embeds into $B_{1} \oplus B_{2}$ with the embedding defined by $1 \mapsto(1,1)$. Then $T Q \cong \underset{p_{i}}{\bigoplus} \mathbb{Z} / p_{i}^{l_{i}-r_{i}} \mathbb{Z}$ uhere $l_{i}=\min \left(m_{i}, n_{i}\right)$.

Proof: We first show that $T Q=\frac{\left\{\left(\frac{a}{b}, \frac{a}{b}\right) ! \frac{a}{b} \in B_{1} \cap B_{2}\right\}}{P}$. Suppose $\left[\left(\frac{a_{1}}{b_{1}}, \frac{a_{2}}{b_{2}}\right)\right]$ is a coset in $T Q$. There must exist an integer $k$ so that $k\left(\frac{a_{1}}{b_{1}}, \frac{a_{2}}{b_{2}}\right) \in$ im $P=\{(x, x) \mid x \in P\}$ (since $1 \mapsto(1,1)$ gives the embedding). Hence $\left(\frac{a_{1}}{b_{1}} ; \frac{a_{2}}{b_{2}}\right)=\left(\frac{x}{k}, \frac{x}{k}\right)$ for some $x \in P$ and $\left[\left(\frac{a_{1}}{b_{1}} ; \frac{a_{2}}{b_{2}}\right)\right] \in \frac{\left\{\left(\frac{a}{b}, \frac{a}{b}\right) \mid \frac{a}{b} \in B_{1} \cap B_{2}\right\}}{P}$. Now assume $\left[\left(\frac{a}{b}, \frac{a}{b}\right)\right]$ is a coset in $\frac{\left\{\left(\frac{a}{b}, \frac{a}{b}\right) ! \frac{a}{b} \in B_{1} \cap B_{2}\right\}}{P}$. Since $b\left(\frac{a}{b}, \frac{a}{b}\right)=(a, a) \in$ $\operatorname{im} P$ in $B_{1} \oplus B_{2},\left[\left(\frac{a}{b}, \frac{a}{b}\right)\right] \in T Q$.

So $T Q=\frac{\left\{\left(\frac{a}{b}, \frac{a}{b}\right) \mid \frac{a}{b} \in B_{1} \cap B_{2}\right\}}{P} \cong \frac{B_{1} \cap B_{2}}{P}$. Now $B_{1} \cap B_{2}=\left\langle\frac{1}{p_{i}^{t_{i}}}\right\rangle$ where $l_{i}=\min \left(m_{i}, n_{i}\right)$. Hence $\frac{B_{2} \cap B_{2}}{P}=\left\langle\frac{1}{p_{i}^{i_{i}}}\right\rangle / P \cong \underset{p_{i}}{\bigoplus} \mathbb{Z} / p_{i}^{l_{i}-r_{i}} \mathbb{Z}$ since a torsion abelian group is the direct sum of its $p_{i}$-torsion subgroups. Note that $l_{2}-r_{2}=\min \left(m_{i}, n_{i}\right)-r_{i} \geq 0$ for all $i$, since $r_{i} \leq m_{i}$ and $r_{i} \leq n_{i}$ for all $i$ by Theorem 2.1 .

Now we use Theorem 3.1 to determine the torsion subgroup resulting from an arbitrary embedding.

Theorem 3.2. Suppose $P$ embeds into $B_{1} \oplus B_{2}$ with the embedding defined by $1 \mapsto\left(\frac{u_{1}}{v_{1}}, \frac{u_{2}}{v_{2}}\right)$. Then $T Q \cong \bigoplus_{p_{i}} \mathbb{Z} / p_{i}^{l_{i}^{\prime}-r_{i}} \mathbb{Z}$ where $l_{i}^{\prime}=\min \left(m_{i}+\right.$ $\left.v_{p_{i}}\left(\frac{u_{1}}{v_{1}}\right), n_{i}+v_{p_{i}}\left(\frac{u_{2}}{v_{2}}\right)\right)$.

Proof: Consider the following commutative diagram

$$
\begin{array}{rlrl}
1 & \mapsto & \left(\frac{u_{1}}{v_{1}}, \frac{u_{2}}{v_{2}}\right) \\
P & \mapsto & B_{1} \oplus B_{2} \\
\| & & \downarrow \downarrow \frac{u_{1}}{u_{3}} \oplus \frac{u_{2}}{u_{2}} \\
P & \mapsto B_{1}^{\prime} \oplus B_{2}^{\prime} \\
1 & \mapsto(1,1)
\end{array}
$$


where $B_{1}^{\prime}=\left\langle\frac{1}{p_{i}^{m}}\right\rangle$ with $m_{i}^{\prime}=m_{i}+v_{p_{i}}\left(\frac{u_{1}}{v_{1}}\right), B_{2}^{\prime}=\left\langle\frac{1}{p_{i}^{n_{i}^{\prime}}}\right\rangle$ with $n_{i}^{\prime}=$ $n_{i}+v_{p_{i}}\left(\frac{u_{2}}{v_{2}}\right)$, and $\frac{v_{k}}{u_{1}} \odot \frac{v_{2}}{u_{2}}: B_{1} \ominus B_{2} \cong B_{1}^{\prime} \oplus B_{2}^{\prime}$ is the isomorphism defined as multiplication by $\frac{v_{1}}{u_{1}}$ in the first coordinate and by $\frac{v_{2}}{u_{2}}$ in the second. Note that $1 \mapsto(1,1)$ does define an embedding of $P$ into $B_{1}^{\prime} \oplus B_{2}^{\prime}$. For, since $1 \mapsto\left(\frac{u_{1}}{v_{1}}, \frac{u_{2}}{v_{2}}\right)$ is an embedding of $P$ into $B_{1} \oplus B_{2}$, $r_{i} \leq m_{i}+v_{p_{T}}\left(\frac{u_{1}}{v_{1}}\right)=m_{i}^{\prime}$ and $r_{i} \leq n_{i}+v_{p_{i}}\left(\frac{u_{2}}{u_{2}}\right)=n_{i}^{\prime}$ for all $i$ by Theorem 2.1. Thus $1 \mapsto(1,1)$ is an embedding of $P$ into $B_{1}^{\prime} \oplus B_{2}^{\prime}$ also by Theorem 2.1. Let $Q^{\prime}=\frac{B_{2}^{\prime} \oplus B_{2}^{\prime}}{P}$ be the quotient resulting from this embedding. Now $Q \cong Q^{\prime}$ since $\frac{v_{1}}{u_{1}} \ominus \frac{v_{2}}{u_{2}}$ induces an isomorphism on the quotients. Hence $T Q \cong T Q^{\prime} \cong \underset{p_{l}}{\bigoplus} \mathbb{Z} / p_{i}^{l_{i}^{\prime}-r_{i}} \mathbb{Z}$ where $l_{i}^{\prime}=\min \left(m_{i}^{\prime}, n_{i}^{\prime}\right)$ by Theorem 3.1 , and the theorem is proved.

The theorems in the remainder of this section are not necessary to prove our eventual result on the existence of completely decomposable extensions in $\operatorname{Ext}(\bar{P}, P)$. However, given that $P, B_{1}$, and $B_{2}$ are groups of pseudo-integers so that it is possible to embed $P$ into $B_{1} \oplus B_{2}$, the theorems provide insight into the naturc of the torsion subgroups of $Q=\frac{B_{1} \oplus B_{2}}{P}$ which occur with respect to different embeddings. For, given one such torsion subgroup, we are able to determine all other torsion subgxoups which it is possible to obtain. We also find, in terms of the exponents of $P, B_{1}$, and $B_{2}$, when it is possible to embed $P$ with a torsionfree quotient. Moreover, we describe under what conditions a given embedding will have a quotient that is torsionfree. Again, we let $P=\left\langle\frac{1}{p_{i}^{\frac{1}{q}}}\right\rangle, B_{1}=\left\langle\frac{1}{p_{i}^{m_{i}}}\right\rangle$, and $B_{2}=\left\langle\frac{1}{p_{i}^{\alpha_{i}}}\right\rangle$ represent the groups of pseudointegers.

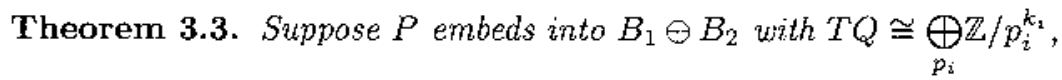
$k_{i} \geq 0$. Let $\bigoplus_{p_{i}} \mathbb{Z} / p_{i}^{k_{i}^{\prime}}$ with $k_{i}^{\prime} \geq 0$ be a torsion group. There exists an embedding of $P$ into $B_{1} \subseteq B_{2}$ so that $T Q \cong \bigoplus_{p_{i}} \mathbb{Z} / p_{i}^{k_{z}^{\prime}}$ if and only if $k_{i}=k_{z}^{\prime}$ almost everywhere.

Proof: Let $1 \mapsto\left(\frac{u_{1}}{v_{1}}: \frac{u_{2}}{v_{2}}\right)$ be an embedding of $P$ into $B_{1} \oplus B_{2}$ so that $T Q \cong \bigoplus_{p_{i}} \mathbb{Z} / p_{i}^{k_{i}} \mathbb{Z}$. We know that $r_{i}-v_{p_{i}}\left(\frac{u_{1}}{v_{1}}\right) \leq m_{i}$ and $r_{i}-v_{p_{i}}\left(\frac{u_{2}}{v_{2}}\right) \leq n_{i}$ for all $i$ (Theorem 2.1). Also, by Theorem $3.2, k_{i}=l_{i}-r_{i}$ whore $l_{i}=$ $\min \left(m_{i}+v_{p_{i}}\left(\frac{u_{1}}{v_{1}}\right), n_{i}+v_{p_{2}}\left(\frac{u_{2}}{v_{2}}\right)\right)$.

Now assume $l \mapsto\left(\frac{a_{1}}{b_{1}}, \frac{a_{2}}{b_{2}}\right)$ is an embedding of $P$ into $B_{1} \oplus B_{2}$ so that $T Q \cong \bigoplus_{p_{i}} \mathbb{Z} / p_{i}^{k_{i}^{\prime}} \mathbb{Z}$. As above, $k_{i}^{\prime}=l_{i}^{\prime}-r_{2}$ where $l_{i}^{\prime}=\min \left(m_{i}+v_{p_{i}}\left(\frac{a_{1}}{b_{1}}\right), n_{i}+\right.$ 
$\left.v_{p_{i}}\left(\frac{a_{2}}{b_{2}}\right)\right)$. Since $v_{p_{i}}\left(\frac{a_{1}}{b_{1}}\right)=v_{p_{i}}\left(\frac{a_{2}}{b_{2}}\right)=v_{p_{i}}\left(\frac{u_{1}}{v_{1}}\right)=v_{p_{i}}\left(\frac{u_{2}}{v_{2}}\right)=0$ for almost all $i, l_{i}=l_{i}^{\prime}=\min \left(m_{i}, n_{i}\right)$ almost everywhere. Hence $k_{i}=k_{i}^{\prime}$ almost everywhere.

Now suppose $k_{i}=k_{i}^{\prime}$ almost everywhere. Let $S_{1}=\left\{i \mid k_{i}^{\prime}>k_{i}\right\}$, $S_{2}=\left\{i \mid k_{i}>k_{i}^{\prime}\right\}, u=\prod_{i \in S_{1}} p_{i}^{k_{i}^{\prime}-k_{i}}$, and $v=\prod_{i \in S_{2}} p_{i}^{k_{i}-k_{i}^{\prime}}$. Note that if $i \in S_{1} \cup S_{2}$ then $v_{p_{i}}\left(\frac{u}{v}\right)=k_{i}^{\prime}-k_{i}$ and otherwise $v_{p_{i}}\left(\frac{u}{v}\right)=0$. We can show that $1 \mapsto\left(\frac{v u_{\lambda}}{v v_{1}}, \frac{v u_{2}}{v v_{2}}\right)$ is an embedding of $P$ into $B_{1} \oplus B_{2}$. For if $i \notin S_{1} \cup S_{2}$, $r_{i}-v_{p_{i}}\left(\frac{u u_{1}}{v v_{1}}\right)=r_{i}-v_{p_{i}}\left(\frac{u_{1}}{v_{1}}\right) \leq m_{i}$ and $r_{i}-v_{p_{i}}\left(\frac{u u_{2}}{v v_{2}}\right)=r_{i}-v_{p_{i}}\left(\frac{v_{i}}{v_{2}}\right) \leq n_{i}$. If $i \in S_{1} \cup S_{2}, r_{i}-v_{p_{1}}\left(\frac{u u_{1}}{v v_{1}}\right)=r_{i}-v_{p_{i}}\left(\frac{u_{1}}{v_{1}}\right)-\left(k_{i}^{\prime}-k_{i}\right)=r_{i}-v_{p_{i}}\left(\frac{u_{1}}{v_{1}}\right)-k_{i}^{\prime}+$ $\min \left(m_{i}+v_{p_{i}}\left(\frac{u_{1}}{v_{1}}\right), n_{i}+v_{p_{i}}\left(\frac{u_{2}}{v_{2}}\right)\right)-r_{i} \leq-v_{p_{i}}\left(\frac{u_{1}}{v_{1}}\right)-k_{i}^{\prime}+m_{i}+v_{p_{i}}\left(\frac{u_{1}}{v_{1}}\right)=m_{i}-$ $k_{i}^{\prime} \leq m_{i}$ as $k_{i}^{\prime} \geq 0$. An analogous argument shows that $r_{i}-v_{p_{i}}\left(\frac{u u_{2}}{v v_{2}}\right) \leq n_{i}$. Hence, we have an embedding by Theorem 2.1.

We see that the torsion subgroup of $Q$ with this embedding is $\bigoplus_{p_{i}} \mathbb{Z} / p_{i}^{k_{i}^{\prime}} \mathbb{Z}$. From Theorem 3.2,TQ $\cong \bigoplus_{p_{i}}^{\mathbb{Z} / p_{i}^{l_{i}^{\prime}}-r_{i}}$ where $l_{i}^{\prime}=\min \left(m_{i}+\right.$ $\left.v_{p_{i}}\left(\frac{u u_{1}}{v v_{1}}\right), n_{i}+v_{p_{i}}\left(\frac{u u_{2}}{v v_{2}}\right)\right)=l_{i}+v_{p_{i}}\left(\frac{u}{v}\right)$. If $i \notin S_{1} \cup S_{2}$, then $l_{i}^{\prime}=l_{i}$ and $l_{i}^{\prime}-r_{i}=l_{i}-r_{i}=k_{i}=k_{i}^{\prime}$. If $i \in S_{1} \cup S_{2}, l_{i}^{\prime}=l_{i}+v_{p_{3}}\left(\frac{u}{v}\right)=l_{i}+\left(k_{i}^{\prime}-k_{i}\right)=$ $k_{i}^{\prime}+r_{i}$ and hence $l_{i}^{\prime}-r_{i}=k_{i}^{\prime}$.

Corollary 3.4. Let $P, B_{1}$, and $B_{2}$ be groups of pseudo-integers so that $P$ embeds into $B_{1} \oplus B_{2}$. Then $T Q$ is finite for all embeddings of $P$ into $B_{1} \oplus B_{2}$ or $T Q$ is infinite for all embeddings of $P$ into $B_{1} \oplus$ $B_{2}$. Moreover, if $T Q$ is always finite then $P$ embeds into $B_{1} \oplus B_{2}$ with torsionfree quotient.

For the next theorems we establish the following notation. Let $\sigma^{+}(P)=\left\{i \mid r_{i}>0\right\}$ and $B_{1} \backslash P=\left\langle\frac{1}{p_{i}^{\pi_{i}}}\right\rangle$ where $s_{i}=\max \left(m_{i}-r_{i}, 0\right)$.

Theorem 3.5. Suppose $P$ embeds into $B_{1} \oplus B_{2}$. There exists an embedding of $P$ into $B_{1} \oplus B_{2}$ so that $Q$ is torsionfree if and only if $\sigma^{+}\left(B_{1} \backslash P\right) \cap \sigma^{+}\left(B_{2} \backslash P\right)$ is finite.

Proof: Suppose $\sigma^{+}\left(B_{1} \backslash P\right) \cap \sigma^{+}\left(B_{2} \backslash P\right)$ is infinite. Let $1 \mapsto\left(\frac{u_{1}}{v_{1}}, \frac{u_{2}}{v_{2}}\right)$ be an arbitrary embedding of $P$ into $B_{1} \oplus B_{2}$. Select $p_{i}$ so that $i \in$ $\sigma^{+}\left(B_{1} \backslash P\right) \cap \sigma^{+}\left(B_{2} \backslash P\right)$ and $v_{p_{i}}\left(v_{1}\right)=v_{p_{i}}\left(v_{2}\right)=0$. Then note that $x=\left(\frac{u_{1}}{v_{1} p_{i}^{r_{i}+1}}, \frac{u_{2}}{v_{2} p_{i}^{r_{i}+1}}\right) \in B_{1} \oplus B_{2}$. Certainly $x \notin \operatorname{im} P \subseteq B_{1} \oplus B_{2}$ but $p_{i} x$ is the image of $\frac{1}{p_{i}^{i}} \in P$ in $B_{1} \oplus B_{2}$. Hence $Q$ has torsion.

Now assume that $\sigma^{+}\left(B_{1} \backslash P\right) \cap \sigma^{+}\left(B_{2} \backslash P\right)$ is finite. Let $S_{1}=\{i \mid$ $r_{i}>m_{i}$ or $\left.r_{i}>n_{i}\right\}$ and note that, by Theorem $2.2, S_{1}$ is finite. Let $S_{2}=\left\{i \mid r_{i}<m_{i}\right.$ and $\left.r_{i}<n_{i}\right\}$ and also note that $S_{2}$ is finite since 
$\sigma^{+}\left(B_{1} \backslash P\right) \cap \sigma^{+}\left(B_{2} \backslash P\right)$ is finite. Now if $i \notin S_{1} \cup S_{2}$ then $r_{i} \leq m_{i}, n_{i}$ and $r_{i}=m_{i}$ or $r_{i}=n_{2}$. Let $1 \mapsto\left(\frac{u_{1}}{v_{1}}, \frac{u_{2}}{v_{2}}\right)$ be an embedding of $P$ into $B_{1} \oplus B_{2}$. By Theorem 3.2, $T Q \cong \underset{p_{i}}{\bigoplus} \mathbb{Z} p_{i}^{l_{i}-r_{2}}$ where $l_{i}=\min \left(m_{i}+v_{p_{i}}\left(\frac{u_{1}}{v_{1}}\right), n_{i}+\right.$ $\left.v_{p_{i}}\left(\frac{u_{2}}{v_{2}}\right)\right)$. Let $S_{3}=\left\{i \mid v_{p_{i}}\left(\frac{u_{1}}{v_{1}}\right) \neq 0\right.$ or $\left.v_{p_{i}}\left(\frac{u_{2}}{v_{2}}\right) \neq 0\right\}$ so that if $i \notin S_{3}$ then $l_{i}=\min \left(m_{i}, n_{i}\right)$. Now if $i \notin S_{1} \cup S_{2} \cup S_{3}$ then $l_{2}=\min \left(m_{i}, n_{i}\right)=r_{i}$ and hence $T Q$ has only a finite number of nontrivial $p$-components. Thus there exists an embedding of $P$ into $B_{1} \oplus B_{2}$ so that the torsion subgroup of the quotient is trivial (Theorem 3.3).

So given that $P$ embeds into $B_{1} \oplus B_{2}$ with torsionfree quotient, we know that $r_{i} \leq m_{i}, n_{i}$ for almost all $i$ and $\sigma^{+}\left(B_{1} \backslash P\right) \cap \sigma^{+}\left(B_{2} \backslash P\right)$ is finite. The following theorem enables us to decide when a given embedding will have a torsionfree quotient in the special case where $r_{i} \leq m_{i}, n_{i}$ for all $i$ and $\sigma^{+}\left(B_{1} \backslash P\right) \cap \sigma^{+}\left(B_{2} \backslash P\right)$ is empty. Although we will not do so explicitly here, we could use a commutative diagram as in the proof of Theorem 3.2 to extend the result to the more general case.

Theorem 3.6. Suppose $P$ embeds into $B_{1} \oplus B_{2}$ with torsionfree quotient. Assume also that $r_{i} \leq m_{i}, n_{i}$ for all $i$ and $\sigma^{+}\left(B_{1} \backslash P\right) \cap \sigma^{+}\left(B_{2} \backslash P\right)$ is empty. Let $1 \mapsto\left(\frac{u_{1}}{v_{1}}, \frac{u_{2}}{v_{2}}\right)$ determine an embedding of $P$ into $B_{1} \oplus B_{2}$. Then $Q$ has torsion if and only if there exists $p_{i}$ so that $p_{i} \mid u_{1}$ or $\gamma_{1}$ holds and $p_{i} \mid u_{2}$ or $\gamma_{2}$ holds where $\gamma_{1}$ is the condition that $i \in \sigma^{+}\left(B_{1} \backslash P\right)$ and $v_{p_{i}}\left(v_{1}\right)<m_{i}-r_{i}, \gamma_{2}$ is the condition that $i \in \sigma^{+}\left(B_{2} \backslash P\right)$ and $v_{p_{i}}\left(v_{2}\right)<n_{i}-r_{i}$.

Now suppose $Q$ has torsion and let $\left[\left(\frac{a_{1}}{b_{1}}, \frac{a_{2}}{b_{2}}\right)\right]$ be a nontrivial cosct of finite order. Hence there exists $k \in \mathbb{Z}(k>1), \frac{c}{d} \in P$, so that $k\left(\frac{a_{1}}{b_{1}}, \frac{a_{2}}{b_{2}}\right)=$ $\left(\frac{c u_{1}}{d v_{1}}, \frac{c u_{2}}{d v_{2}}\right) \in B_{1} \oplus B_{2}$. So $\frac{a_{1}}{b_{1}}=\frac{c u_{1}}{k d v_{1}}$ and $\frac{a_{2}}{b_{2}}=\frac{c u_{2}}{k d v_{2}}$, where we may assume $\frac{c}{k d}$ to be reduced (if it were not, we would replace $\frac{c}{k d}$ with its reduced form). Since our chosen coset was nontrivial, $\frac{c}{k d} \notin P$ and hence there exists $p_{i}$ so that $v_{p_{i}}(k d)>r_{i}$. Now $\left(\frac{a_{1}}{b_{1}}, \frac{a_{2}}{b_{2}}\right)=\left(\frac{c u_{1}}{k d v_{1}}, \frac{c u_{2}}{k d v_{2}}\right) \in B_{1} \oplus B_{2}$ and $\sigma^{+}\left(B_{1} \backslash P\right) \cap \sigma^{+}\left(B_{2} \backslash P\right)$ is cmpty so $p_{i} \mid u_{1}$ or $p_{i} \mid u_{2}$. We assume $p_{i} \mid u_{1}$ and note that if $p_{i} \mid u_{2}$ also, there is nothing more to prove. Otherwise, since $v_{p_{i}}(k d)>r_{i}$ and $\frac{c u_{2}}{k d v_{2}} \in B_{2}$, we have $i \in \sigma^{+}\left(B_{2} \backslash P\right)$ and $v_{p_{i}}\left(v_{2}\right)<n_{i}-r_{i}$, i.c., we have condition $\gamma_{2}$. Similarly, if we had assurned $p_{\imath} \mid u_{2}$ then we could conclude that $p_{i} \mid u_{1}$ or $\gamma_{1}$ is true.

Let $p_{i}$ be a prime so that $p_{i} \mid u_{1}$ or $\gamma_{1}$ holds and $p_{i} \mid u_{2}$ or $\gamma_{2}$ holds. If $p_{i} \mid u_{1}$ and $p_{i} \mid u_{2}$ then $u_{1}=p_{i} u_{1}^{\prime}$ and $u_{2}=p_{i} u_{2}^{\prime}$. Now $x=\left(\frac{u_{i}^{\prime}}{p_{i}^{\prime \prime} v_{1}}, \frac{u_{i}^{\prime}}{p_{i}^{\prime i} v_{2}}\right) \in B_{1} \oplus B_{2}$ since we assume $\frac{u_{1}}{v_{1}}, \frac{u_{2}}{v_{2}}$ are reduced and $r_{i} \leq m_{i}, n_{i}$ for all $i$. Note that $x \notin \operatorname{im} P$ in $B_{1} \oplus B_{2}$ but $p_{i} x$ is the image of $\frac{1}{p_{i}^{12}}$ in $B_{1} \oplus B_{2}$. Thus $[x]$ is a nontrivial torsion element in $Q$. 
Now assume $p_{i} \mid u_{1}$ and $\gamma_{2}$ holds and again let $u_{1}=p_{i} u_{1}^{\prime}$. Observe that $x=\left(\frac{u_{1}^{\prime}}{p_{i}^{\alpha_{2}} v_{3}}, \frac{u_{2}}{p_{i}^{r_{i}+1} v_{2}}\right) \in B_{1} \oplus B_{2}$ for as above $\frac{u_{1}^{\prime}}{p_{i}^{y_{2}} v_{1}} \in B_{1}$ and $\gamma_{2}$ ensures that $\frac{u_{3}}{p_{i}^{r_{i}+1} v_{2}} \in B_{2}$. Also as above $[x]$ is an element of order $p_{i}$ in $Q$. A symmetric argument shows that $Q$ has torsion if we assume condition $\gamma_{1}$ and that $p_{i} \mid u_{2}$. Since $\sigma^{+}\left(B_{1} \backslash P\right) \cap \sigma^{+}\left(B_{2} \backslash P\right)$ is empty the case where both $\gamma_{1}$ and $\gamma_{2}$ hold cannot occur.

\section{Torsionfree Quotients.}

We are almost ready to describe when $\operatorname{Ext}(\ddot{P}, P)$ contains nontrivial completely decomposable extensions. Recall that this means that we seek the existence of groups of pseudo-integers $B_{1}$ and $B_{2}\left(P \not \equiv B_{1}\right.$ or $\bar{P} \not B_{2}$ and $P \not B_{2}$ or $\bar{P} \not B_{1}$ ) so that $P$ embeds into $B_{1} \oplus B_{2}$ with quotient isomorphic to $\bar{P}$. Hence, given that $P$ embeds into $B_{1} \oplus B_{2}$, we now characterize the torsionfree quotient of $Q=\frac{B_{1} \oplus B_{2}}{P}$, denoted $Q / T Q$, with respect to any embedding. This allows us, in the particular case when the torsion subgroup $T Q$ is trivial, to decide if $Q$ is isomorphic to $\bar{P}$. Again we represent our groups of pseudo-integers by $P=\left\langle\frac{1}{p_{i}^{2 i}}\right\rangle$, $B_{1}=\left\langle\frac{1}{p_{i}^{m_{i}}}\right\rangle$, and $B_{2}=\left\langle\frac{1}{p_{i}^{h_{i}}}\right\rangle$.

Theorem 4.1. Suppose that $P, B_{1}$, and $B_{2}$ are groups of pseudointegers so that $P$ embeds into $B_{1} \oplus B_{2}$ with quotient $Q$. Then the torsionfree quotient of $Q$, denoted $Q / T Q$, with respect to any embedding of $P$ into $B_{1} \oplus B_{2}$ is isomorphic to $\left\langle\frac{1}{p_{i}^{s_{i}}}\right\rangle$ where $s_{i}=\max \left(m_{i}, n_{i}\right)$.

Proof: Since $P$ embeds into $B_{1} \oplus B_{2}$, we know that $r_{i} \leq m_{i}, n_{i}$ for almost all $i$ by Theorem 2.2. Our argument considers the two cascs that $r_{i} \leq m_{i}, n_{i}$ for all $i$ and $r_{i} \leq m_{i}, n_{2}$ for all $i$. We suppose first that $r_{i} \leq m_{i}, n_{i}$ for all $i$ so that $1 \mapsto(1,1)$ determines an embedding of $P$ into $B_{1} \oplus B_{2}$ (Theorem 2.1). By the proof of Theorem 3.1, we know that $T Q$ with respect to this embedding is $\frac{\left\{\left(\frac{a}{b}, \frac{a}{b}\right) \mid \frac{a}{b} \in B_{1} \cap B_{2}\right\}}{P}$ and

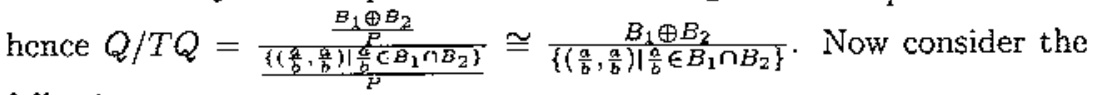
following sequence

$$
\left\{\left(\frac{a}{b}, \frac{a}{b}\right) \mid \frac{a}{b} \in B_{1} \cap B_{2}\right\} \stackrel{i}{\rightarrow} B_{1} \oplus B_{2} \stackrel{\pi}{\rightarrow}\left\langle\frac{1}{p_{i}^{s_{i}}}\right\rangle
$$

where $i$ is inclusion, $\pi$ is defined by $\pi\left(\frac{a_{2}}{b_{1}}, \frac{a_{2}}{b_{2}}\right)=\frac{a_{1}}{b_{1}}-\frac{a_{2}}{b_{2}}$, and $s_{i}=$ $\max \left(m_{i}, n_{i}\right)$. We see that the sequence is exact. First we note that im $i \subseteq$ ker $\pi$. Now suppose $\left(\frac{a_{1}}{b_{1}}, \frac{a_{2}}{b_{2}}\right) \in$ ker $\pi$. Then $\frac{a_{3}}{b_{1}}-\frac{a_{2}}{b_{2}}=0$ which implies $\frac{a_{1}}{b_{1}}=\frac{a_{2}}{b_{2}}$ and hence that $\left(\frac{a_{1}}{b_{1}}, \frac{a_{2}}{b_{2}}\right) \in \operatorname{im} i$. Finally, we show that $\pi$ 
is onto. Let $\frac{1}{p_{i}^{s_{i}}}$ be an arbitrary generator of $\left\langle\frac{1}{p_{i}^{s_{i}}}\right\rangle$. Now $s_{i}=\max \left(m_{i}, n_{i}\right)$ and so if $s_{i}=m_{i}$ then $\pi\left(\frac{1}{p_{i}^{m_{i}}}, 0\right)=\frac{1}{p_{i}^{s_{3}}}$. Otherwise $\left(0,-\frac{1}{p_{i}^{n_{i}}}\right)=\frac{1}{p_{i}^{s_{i}}}$. Our sequence is thus short exact and $Q / T Q \cong \frac{B_{1} \oplus B_{2}}{\left\{\left(\frac{a}{b}, \frac{a}{b}\right) \mid \frac{a}{b} \in B_{1} \cap B_{2}\right\}} \cong\left\langle\frac{1}{p_{i}^{s_{i}}}\right\rangle$.

We now assume that $r_{i} \& m_{2}, n_{i}$ for all $i$ and we let $1 \mapsto\left(\frac{u_{1}}{v_{1}}, \frac{u_{2}}{v_{2}}\right)$ determine an arbitrary embedding of $P$ into $B_{1} \oplus B_{2}$. Consider the following commutative diagram

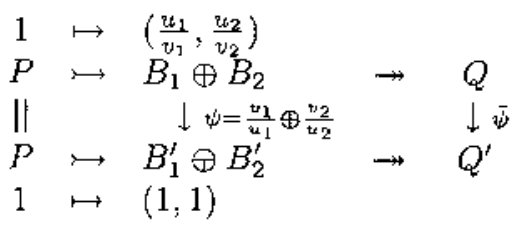

where $B_{1}^{\prime}=\left\langle\frac{1}{p_{i}^{m_{i}^{\prime}}}\right\rangle$ with $m_{i}^{\prime}=m_{i}+v_{p_{i}}\left(\frac{u_{1}}{v_{1}}\right), B_{2}^{\prime}=\left\langle\frac{1}{p_{i}^{n_{i}^{\prime}}}\right\rangle$ with $n_{i}^{\prime}=$ $n_{i}+v_{p_{i}}\left(\frac{u_{2}}{v_{2}}\right), Q=\frac{B_{1} \oplus B_{z}}{P}, Q^{\prime}=\frac{B_{1}^{\prime} \oplus R_{2}^{\prime}}{P}, \psi$ is the isomorphism defined by multiplication by $\frac{v_{1}}{u_{1}}$ in the first coordinate and by $\frac{v_{2}}{u_{2}}$ in the second, and $\bar{\psi}$ is the isomorphism induced by $\psi$ on the quotients. We note that $1 \mapsto(l, 1)$ is an embedding of $P$ into $B_{1}^{\prime} \oplus B_{2}^{\prime}$. For, since $1 \mapsto\left(\frac{u_{1}}{v_{1}}, \frac{u_{2}}{v_{2}}\right)$ is an embedding of $P$ into $B_{1} \oplus B_{2}, r_{i}-v_{p_{i}}\left(\frac{u_{1}}{v_{1}}\right) \leq m_{i}$ and $r_{2}-v_{p_{i}}\left(\frac{\underline{u}_{2}}{v_{2}}\right) \leq n_{i}$ for all $i$ by Theorem 2.1. Hence $r_{i} \leq m_{i}+v_{p_{i}}\left(\frac{u_{\mathcal{A}}}{v_{1}}\right)=m_{i}^{\prime}$ and $r_{i} \leq$ $n_{i}+v_{p_{i}}\left(\frac{u_{2}}{v_{2}}\right)=n_{i}^{\prime}$ for all $i$ and again by theorem $2.1,1 \mapsto(1,1)$ is an embedding of $P$ into $B_{1}^{\prime} \oplus B_{2}^{\prime}$.

Since $\bar{\psi}$ is an isomorphism from $Q$ to $Q^{\prime}, \bar{\psi}$ restricts to $\psi^{\prime}: T Q \cong T Q^{\prime}$. Thus we have the following commutative diagram

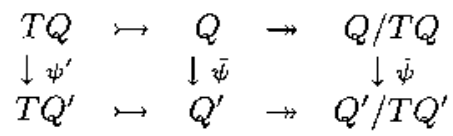

where $\hat{\psi}$ is the isomorphism induced by $\bar{\psi}$ on the torsionfree quotients. Now $P$ embeds into $B_{1}^{\prime} \oplus B_{2}^{\prime}$ by $1 \mapsto(1,1)$ so by an argument identical to the above $Q^{\prime} / T Q^{\prime} \cong\left\langle\frac{1}{p_{i}^{s_{i}}}\right\rangle$ where $s_{i}^{\prime}=\max \left(m_{i}^{\prime}, n_{i}^{\prime}\right)=\max \left(m_{i}+\right.$ $\left.v_{p_{i}}\left(\frac{u_{1}}{v_{1}}\right), n_{1}+v_{p_{\mathrm{t}}}\left(\frac{u_{2}}{v_{2}}\right)\right)$. Let $s_{i}=\max \left(m_{i}, n_{i}\right)$ and note that $s_{i}=s_{i}^{\prime}$ almost everywhere. Hence $\left\langle\frac{1}{p_{i}^{s_{i}}}\right\rangle \cong\left\langle\frac{1}{p_{i}^{s_{2}}}\right\rangle$ and thus $Q / T Q \cong Q^{\prime} / T Q^{\prime} \cong$ $\left\langle\frac{1}{p_{i}^{s_{i}}}\right\rangle \cong\left\langle\frac{1}{p_{i}^{s_{i}}}\right\rangle$ where $s_{i}=\max \left(m_{i}, n_{i}\right)$. We remark that if $P$ embeds into $B_{1} \oplus B_{2}$ with torsionfree quotient then $Q \cong\left\langle\frac{1}{p_{i}^{s_{3}}}\right\rangle$. 
All the theorems in Sections 2 and 3 gencralize in the obvious ways to the case where $P$ is embedded into $\bigoplus_{j=1}^{k} B_{j}, B_{j}=\left\langle\frac{1}{p_{i} m_{i j}}\right\rangle$, the direct sum of $k$ groups of pseudo-integers for $k>2$. However, we see that Theorem 4.1 does not generalize. For, we might expect that if $P$ embeds into $\bigoplus_{j=1}^{k} B_{j}$ with quotient $Q=\bigoplus_{j=1}^{k} B_{j} / P$ then the torsionfree quotient $Q / T Q$ would be isomorphic to $\bigoplus_{j=1}^{k-1} B_{j}^{\prime}$ where $B_{j}^{\prime}=\left\langle\frac{1}{p_{i}^{s_{j}}}\right\rangle, s_{i j}=$ $\max \left(m_{i j}, m_{i_{j+1}}\right)$. But this is not always true. It is certainly the case that $T Q \cong \frac{\left\{\left(\frac{a}{b}, \frac{a}{b}, \cdots, \frac{a}{b}\right) \mid \frac{a}{b} \in \bigcap_{j=1}^{k} B_{j}\right\}}{B}$ and hence $Q / T Q \cong \frac{\bigoplus_{j=1}^{k} B_{j}}{\left.\left\{\left(\frac{a}{b}, \frac{a}{b}, \cdots, \frac{a}{b}\right)\right\} \frac{a}{b} \in \bigcap_{j=1}^{k} B_{j}\right\}}$.

Further, we can show that the following sequence is exact:

$$
\left\{\left(\frac{a}{b}, \frac{a}{b}, \cdots, \frac{a}{b}\right) \mid \frac{a}{b} \in \bigcap_{j=1}^{k} B_{j}\right\} \stackrel{i}{\mapsto} \bigoplus_{j=1}^{k} B_{j} \stackrel{\pi}{\rightarrow} \bigoplus_{j=1}^{k-1} B_{j}^{\prime}
$$

where $i$ is inclusion map and $\pi: \bigoplus_{j=1}^{k} B_{j} \rightarrow \bigoplus_{j=1}^{k-1} B_{j}^{\prime}$ is defined by $\pi\left(b_{1}, b_{2}, \cdots, b_{k}\right)=\left(b_{1}-b_{2}, b_{2}-b_{3}, \cdots, b_{k-1}-b_{k}\right)$. However, it is not in general true that $\pi$ is onto. For suppose for some $p_{i_{1}}, m_{i_{1}}=1, m_{i_{2}}=2$, $m_{i_{3}}=1$ so that $\max \left(m_{i_{1}}, m_{i_{2}}\right)=2$ and $\max \left(m_{i_{2}}, m_{i_{3}}\right)=2$. It is not possible to find an element in $\bigoplus_{j=1}^{k} B_{j}$ which goes to $\left(\frac{1}{p_{i}^{2}}, \frac{1}{p_{i}^{2}}, 0, \cdots, 0\right)$ under $\pi$. So we know only that $Q / T Q \cong \operatorname{im} \pi=\left\langle\left(b_{1}-b_{2}, b_{2}-b_{3}, \cdots, b_{k-1}-b_{k}\right)\right|$ $\left.b_{i} \in B_{i}\right\rangle$ in $\bigoplus_{j=1}^{k-1} B_{j}^{\prime}$.

We now determine when $\operatorname{Ext}(\bar{P}, P)$ contains nontrivial completely decomposable extensions.

Theorem 4.2. Let $\bar{P}=\left\langle\frac{1}{p_{i}^{r_{i}}}\right\rangle$ and $P=\left\langle\frac{1}{p_{i}^{2_{i}}}\right\rangle$ be groups of pseudointegers. Then $\operatorname{Ext}(\bar{P}, P)$ contains nontrivial completely decomposable extensions if and only if $r_{i} \leq \bar{r}_{i}$ almost everywhere and $r_{i}<\bar{r}_{i}$ for an infinite number of primes. 
Proof: Suppose $\operatorname{Ext}(\bar{P}, P)$ contains nontrivial completely decomposable extensions. Let $P \hookrightarrow B_{1} \oplus B_{2} \rightarrow \bar{P}$ be such an extension where $B_{1}=$ $\left\langle\frac{1}{p_{i}^{n_{i}}}\right\rangle, B_{2}=\left\langle\frac{1}{p_{i}^{n_{2}}}\right\rangle$, and $1 \mapsto\left(\frac{u_{3}}{v_{1}}, \frac{u_{2}}{v_{2}}\right)$ determines the embedding of $P$ into $B_{1} \oplus B_{2}$. Since $Q=\frac{B_{1} \ominus B_{2}}{P}$ is isomorphic to $\bar{P}, Q$ is torsionfree. By Theorcm $3.2, T Q \cong \bigoplus_{p_{2}} \mathbb{Z} / p_{i}^{l_{i}-r_{i}} \mathbb{Z}$ where $l_{i}=\min \left(m_{i}+v_{p_{2}}\left(\frac{u_{1}}{v_{1}}\right), n_{i}+v_{p_{i}}\left(\frac{u_{2}}{v_{2}}\right)\right)$. Hence $l_{i}=r_{i}$ for all $i$. Let $S_{1}=\left\{i \mid v_{p_{i}}\left(\frac{u_{1}}{v_{1}}\right) \neq 0\right.$ or $\left.v_{p_{i}}\left(\frac{u_{2}}{v_{2}}\right) \neq 0\right\}$ and note that $S_{1}$ is finite. For $i \notin S_{1}, l_{i}=\min \left(m_{i}, n_{i}\right)$. Now by Theorem 4.1, $Q / T Q \cong Q \cong\left\langle\frac{1}{p_{i}^{s_{i}}}\right\rangle$ where $s_{i}=\max \left(m_{i}, n_{i}\right)$. So $\bar{P} \cong\left\langle\frac{1}{s_{i}^{s_{i}}}\right\rangle$ which implies that $\bar{r}_{i}=s_{i}$ for almost all $i$. Let $S_{2}=\left\{i \mid \bar{r}_{i} \neq s_{i}\right\}$. For $i \notin S_{1} \cup S_{2}$ (a finite set), $r_{i}=\min \left(m_{i}, n_{i}\right) \leq \max \left(m_{i}, n_{i}\right)=s_{2}=\bar{r}_{i}$. Suppose $r_{i}<\bar{r}_{i}$ for only a finite number of primes and let $S_{3}=\left\{i \mid r_{i}<\ddot{r}_{i}\right\}$. For $i \notin S_{1} \cup S_{2} \cup S_{3}$ (a finite set), $r_{i}=\min \left(m_{i}, n_{i}\right)=\max \left(m_{i}, n_{i}\right)=\bar{r}_{i}$. Hence $m_{i}=n_{i}=r_{i}=\bar{r}_{i}$ and $P \cong \bar{P} \cong B_{1} \cong B_{2}$ which contradicts our assumption of nontriviality.

Now suppose $r_{i} \leq \bar{r}_{i}$ almost everywhere and $r_{i}<\bar{r}_{i}$ for an infinite number of primes. We may assume without loss of generality that $r_{i} \leq \bar{r}_{i}$ everywhere. For, since $r_{i} \leq \bar{r}_{2}$ for all but a finite number of primes, we could replace $P$ with an isomorphic group $P^{\prime}=\left\langle\frac{1}{p_{i}^{r_{i}}}\right\}$ so that $r_{i}^{\prime} \leq r_{i}$ for all primes. Now let $S=\left\{i \mid r_{i}<\bar{r}_{i}\right\}$ and $\bar{S}=\left\{i \mid r_{i}=\bar{r}_{i}\right\}$. Divide $S$ into two disjoint infinite subsets $S_{1}$ and $S_{2}$. Let $B_{1}=\left\langle\frac{1}{p_{i}^{n_{i}}}\right\rangle$ where $m_{i}=$ $\left\{\begin{array}{ll}r_{i} & i \in S_{1} \\ \bar{r}_{i} & i \in S_{2} \cup \bar{S}\end{array}\right.$ and $B_{2}=\left\langle\frac{1}{p_{i}^{n_{i}}}\right\rangle$ where $n_{i}=\left\{\begin{array}{ll}r_{i} & i \in S_{2} \cup \bar{S} \\ \bar{r}_{i} & i \in S_{1}\end{array}\right.$. Now $1 \mapsto(1,1)$ determines an embedding of $P$ into $B_{1} \oplus B_{2}$ since $r_{i} \leq m_{i}, n_{i}$

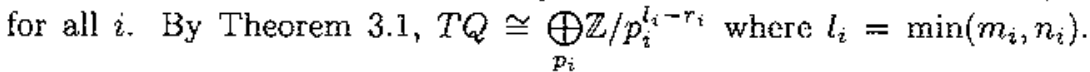
But $\min \left(m_{i}, n_{i}\right)=\min \left(r_{i}, \bar{r}_{i}\right)=r_{i}$ since $r_{i} \leq \bar{r}_{i}$ for all $i$ and thus $T Q$ is trivial. According to Theorem $4.1, Q / T Q \cong Q \cong\left\langle\frac{1}{p_{2}^{s_{2}}}\right\rangle$ where $s_{i}=$ $\max \left(m_{i}, n_{2}\right)$. But $\max \left(m_{i}, n_{i}\right)=\max \left(r_{i}, \bar{r}_{i}\right)=\bar{r}_{i}$. Hence $Q \cong\left\langle\frac{1}{p_{i}^{s_{i}}}\right\rangle=\bar{P}$. Now $P \not B_{1}, P \not B_{2}, \bar{P} ¥ B_{1}$, and $\bar{P} \not B_{2}$ since $S_{1}$ and $S_{2}$ are infinite sets. Thus $P \mapsto B_{1} \oplus B_{2} \rightarrow \bar{P}$ with the embedding $1 \mapsto(1,1)$ is a nontrivial completely decomposable extension of $P$ by $\bar{P}$.

We can now prove the following theorem which will allow us to simplify our notion of nontrivial extension. We again assume $P=\left\langle\frac{1}{p_{i}^{i}}\right\rangle, \bar{P}=$ $\left\langle\frac{1}{p_{i}^{i_{i}}}\right\rangle, B_{1}=\left\langle\frac{1}{p_{i}^{m_{i}}}\right\rangle$, and $B_{2}=\left\langle\frac{1}{p_{i}^{n_{i}}}\right\rangle$ are groups of pseudo-integers.

Theorem 4.3. Suppose $P \neg B_{1} \oplus B_{2} \rightarrow \bar{P}$ is exact. Then $P \cong B_{1}$ if and only if $\bar{P} \cong B_{2}$. 
Proof: We see, from the Proof of Theorem 4.2, that $r_{i}=\min \left(m_{i}, n_{i}\right)$ almost everywhere. By Theorem 4.1, $\bar{r}_{i}=\max \left(m_{i}, n_{i}\right)$ almost everywhere. Hence $r_{i}=m_{i}$ almost cverywhere if and only if $m_{i} \leq n_{i}$ almost everywhere if and only if $\bar{r}_{i}=n_{i}$ almost everywhere.

We could just as easily prove that if $P \mapsto B_{1} \oplus B_{2} \rightarrow \ddot{P}$ is exact then $P \cong B_{2}$ if and only if $\vec{P} \cong B_{1}$. These results do allow us to make our definition of nontrivial extension more concise. We had previously defined an extension $P \longmapsto B_{1} \oplus B_{2} \rightarrow \bar{P}$ to be trivial if $P \cong B_{1}, \bar{P} \cong B_{2}$ or $P \cong B_{2}, \bar{P} \cong B_{1}$ and nontrivial otherwise. With regard to the above, such an extension is now trivial if $P \cong B_{1}$ or $P \cong B_{2}$ and hence nontrivial if $P \not B_{1}$ and $P \not B_{2}$. We note that we could just as easily use $\vec{P}$ in place of $P$ in our new definition.

In [4] it is shown that if $\bar{P}=\left\langle\frac{1}{p_{i}^{\tau_{i}}}\right\rangle$ and $P=\left\langle\frac{1}{p_{i}^{p_{i}}}\right\rangle$ are groups of pscudointegers so that $r_{i}<\bar{r}_{i}$ for an infinite number of $i$ then $\operatorname{Ext}(\dot{P}, P) \mathrm{con}-$ tains extensions $P \mapsto E \rightarrow \bar{P}$ where $E$ is indecomposable as a pseudofrce group of rank 2. So, referring to Theorem 4.2, we sec that if $\operatorname{Ext}(\tilde{P}, P)$ contains extensions which are completely decomposable it must also contain those which are not. Further, we can use the theorems in Section 1 to describe the abelian group structures of $\operatorname{Hom}(\bar{P}, P)$ and $\operatorname{Ext}(\bar{P}, P)$ for the case when $\operatorname{Ext}(\bar{P}, P)$ contains completely decomposable extensions, i.e. when $r_{i} \leq \dot{r}_{i}$ almost everywhere and $r_{i}<\bar{r}_{i}$ for an infinite number of $i$. Since $r_{i}$ must be less than $\bar{r}_{i}$ for infinitely many $i$, we see that $\operatorname{Hom}(\bar{P}, P)$ must be trivial and hence $\operatorname{Ext}(\bar{P}, P) \cong V \oplus(\mathbb{Q} / \mathbb{R})$ where $V$ is a $Q$-vector space of rank $c$. It seems that we should be able to determine where the completely decomposable extensions are to be found in this structure. Do they correspond to torsion elements, infinite elements, mixed elements, or some combination of these? We may also ask a similar question for the indecomposable extensions.

\section{References}

1. C. Casacuberta and P. Hilton, On the extended genus of finitely generated abelian groups, Bull. Soc. Mat. Belg. (Serie A) Tome XLI, 1 (1989), 51-72.

2. P. Hilton, On groups of pseudo-integers, Acta Math. Sinica 4, 2 (1988), 189-192.

3. P. Hilton and U. Stammbach, "A course in homological algebra," Springer-Verlag; 1971.

4. R. Militello and H. Ries, On pseudofree groups and sequential representations, to be submitted. 
5. H. RiES, On the calculation of a certain extension group, to appear in Bull. Soc. Mat. Belg.

Department of Mathernatics

East Carolina University

Greenville, North Carolina

U.S.A.

Rebut el 24 de Maig de 1993 